\title{
A retrospective comparison of outcomes for open vs. laparoscopic surgical techniques in pediatric ulcerative colitis
}

\author{
Brent A. Willobee ${ }^{1}$, Jennifer A. Nguyen ${ }^{1}$, Anthony Ferrantella ${ }^{1}$, Hallie J. Quiroz ${ }^{1}$, Anthony R. Hogan ${ }^{1}$, \\ Ann-Christina Brady ${ }^{1}$, Samir Pandya ${ }^{2}$, Amber H. Langshaw ${ }^{3}$, Juan E. Sola ${ }^{1}$, Chad M. Thorson ${ }^{1}$, \\ Eduardo A. Perez ${ }^{1}$
}

${ }^{1}$ Daughtry Family Department of Surgery, University of Miami Miller School of Medicine, Miami, FL, USA; ${ }^{2}$ Department of Surgery, UT Southwestern Medical Center, Dallas, TX USA; ${ }^{3}$ Department of Pediatrics, University of Miami Miller School of Medicine, Miami, FL, USA Contributions: (I) Conception and design: EA Perez, CM Thorson, BA Willobee, JA Nguyen; (II) Administrative support: None; (III) Provision of study materials or patients: None; (IV) Collection and assembly of data: EA Perez, CM Thorson, BA Willobee, JA Nguyen; (V) Data analysis and interpretation: EA Perez, CM Thorson, BA Willobee; (VI) Manuscript writing: All authors; (VII) Final approval of manuscript: All authors.

Correspondence to: Eduardo A. Perez, MD, MSPH. Daughtry Family Department of Surgery, University of Miami Miller School of Medicine, Miami, FL, USA. Email: eperez3@med.miami.edu.

Background: Ulcerative colitis (UC) is an aggressive disease in the pediatric population and a cause
of significant, lifelong morbidity. The aim of this study is to compare surgical complications in pediatric
patients undergoing laparoscopic vs. open surgical treatment for UC.
Methods: We queried the Kids' Inpatient Database (KID) for all cases of UC undergoing surgical
treatment in 2009 and 2012 . We identified patients who received total colectomy without proctectomy
(n=413) or total proctocolectomy ( $\mathrm{n}=196)$ and performed univariate and multivariate analyses comparing
laparoscopic vs. open procedures. Results: In pediatric UC patients undergoing total colectomy without proctectomy, open procedures were associated with more complications than laparoscopic, including fluid and electrolyte disorders $(40 \%$ vs. $28 \%$ ), surgical wound dehiscence (6\% vs. $2 \%)$, septicemia (18\% vs. $2 \%)$, and gastrointestinal disorders $(16 \%$ vs. $7 \%$ ) among others, all $\mathrm{P}<0.05$. Likewise, in patients with $\mathrm{UC}$ undergoing total proctocolectomy, there were more complications in open vs. laparoscopic technique, including increased transfusion requirements ( $25 \%$ vs. $7 \%, \mathrm{P}=0.001)$ and significantly more gastrointestinal upset, including nausea, vomiting, and diarrhea ( $11 \%$ vs. $1 \%, \mathrm{P}=0.003)$. In multivariate analysis, patients who underwent total colectomy with or without proctectomy had an increased risk of experiencing any complication when their procedure was performed in an open or non-elective fashion (all odds ratio $>2.4$; all $\mathrm{P}<0.001$ ).

Conclusions: The laparoscopic approach was associated with significantly lower rates of surgical complications in pediatric patients undergoing total colectomy with or without proctectomy for UC. These findings demonstrate that laparoscopic technique compares favorably, and may be preferable, to the open approach in selected pediatric patients with UC.

Keywords: Laparoscopic; outcomes; inflammatory bowel disease (IBD); minimally invasive; ulcerative colitis (UC); pediatric surgery; minimally invasive surgery (MIS)

Received: 16 April 2020; Accepted: 20 June 2020; Published: 25 July 2021.

doi: $10.21037 /$ tgh-20-189

View this article at: http://dx.doi.org/10.21037/tgh-20-189

^, ORCID: 0000-0001-8480-5428. 


\section{Introduction}

Ulcerative colitis (UC), a type of inflammatory bowel disease (IBD), is a chronic disease that can lead to significant lifelong morbidity $(1,2)$. It has been estimated that up to $25 \%$ of new cases are diagnosed in the pediatric population $(3,4)$. Among those diagnosed with UC, $20-30 \%$ will require major surgery over their lifetime and over $50 \%$ will require surgery within five years of diagnosis $(5,6)$. The most common indications for surgery include severe colitis refractory to medical management, failure to thrive, colonic dysplasia, and malignancy $(5,7)$.

Medical management is the mainstay for treatment, but surgical intervention plays a role in the more advanced or severe presentations. It is often reserved for those who are critically ill or are refractory to medical management, resulting in a high proportion of urgent or emergent cases (8). For those requiring surgery, the two most common surgical procedures in the US are total colectomy and total proctocolectomy (9). Despite recent advances in minimally invasive techniques, a significant number of these operations are still being performed in an open fashion (10).

In 2015, a retrospective analysis using the ACS NSQIP pediatric database compared surgical outcomes and postoperative length of stay (LOS) between laparoscopic $(n=103)$ versus open $(n=37)$ colectomy in a cohort of pediatric patients with UC. This study was able to demonstrate equivalent outcomes between the two methods. However, the authors felt their study was underpowered to detect a difference in surgical complications between the two cohorts (6). Many additional studies have demonstrated superior results in terms of outcomes when laparoscopy is employed, but these have largely been single institution studies or a single surgeon experience $(11,12)$. The aim of our study is to provide a comprehensive analysis of surgical outcomes in pediatric patients undergoing laparoscopic and open surgical treatment for UC. We hypothesized that the laparoscopic approach in pediatric UC patients is significantly associated with a decrease in both postoperative length of stay as well as overall surgical complication rates. We present the following article in accordance with the STROBE reporting checklist (available at http://dx.doi. org/10.21037/tgh-20-189).

\section{Methods}

Nationwide data on U.S. children with UC that underwent surgical treatment in the years 2009 and 2012 were obtained from the Agency for Healthcare Research and Quality (AHRQ)-sponsored Healthcare Cost and Utilization Project (HCUP) Kids' Inpatient Database (KID) (13). The KID is the largest publicly available all-payer pediatric inpatient care database in the U.S. and is released on a three-year cycle. It contains data from approximately 3 million pediatric discharges each year across 44 different states and is weighted for national estimates of roughly 7 million hospitalizations. Therefore, the KID is a powerful tool to analyze national trends in healthcare utilization, access, charges, quality, and outcomes for both common and rare pediatric conditions. The Institutional Review Board of the University of Miami waived the requirement for approval of this study since the KID contains de-identified, publicly available data.

Children (age $\leq 20$ years) with a diagnosis of UC were identified in the KID by the International Classification of Disease, Ninth Revision, Clinical Modification (ICD9-CM) diagnosis code(s) 556.0, 556.1, 556.2, 556.3, 556.6, 556.8, and 556.9. Patients who underwent an operation for colectomy or proctocolectomy were identified with the ICD-9-CM procedure codes 45.62, 45.81, 45.82, $46.20,46.21$, and 46.23 which differentiate between procedures performed by an open or laparoscopic approach. Patient status (elective $v s$. non-elective) was determined at the time of admission. Patients with both an open and laparoscopic approach were excluded as well as patients with a concurrent diagnosis code for colorectal malignancy. Nationwide population-based incidences were estimated from KID using the weight values provided in the data set for each case. Denominators for calculation of incidences were taken from the United States Census Bureau website (14).

The patient demographics, concurrent diagnoses, frequency of complications, length of stay, and treating hospital characteristics were compared. Categorical variables were compared using a chi-squared test, and continuous variables were compared using Student's $t$-test. Significance was set at $\mathrm{P}<0.05$. Multivariate regression models were constructed for identification of independent predictors of mortality. Variables with more than $20 \%$ missing data were not analyzed in the multivariate analysis. Results were weighted for national estimates according to HCUP standards. Statistical analyses were performed using SPSS Statistics version 24, IBM Corporation, Armonk, New York. 
Table 1 Demographics

\begin{tabular}{|c|c|c|}
\hline \multirow{2}{*}{ Characteristic } & \multicolumn{2}{|c|}{ Total } \\
\hline & $\mathrm{N}$ & $\%$ \\
\hline Total & 609 & 100 \\
\hline \multicolumn{3}{|l|}{ Type of admission } \\
\hline Elective & 300 & 49 \\
\hline Non-elective & 309 & 51 \\
\hline \multicolumn{3}{|l|}{ Gender } \\
\hline Female & 295 & 48 \\
\hline Male & 314 & 52 \\
\hline \multicolumn{3}{|l|}{ Race } \\
\hline Caucasian & 399 & 66 \\
\hline African American & 38 & 6 \\
\hline Hispanic & 57 & 9 \\
\hline Native American & \# & $<1$ \\
\hline Other & 23 & 4 \\
\hline Unknown & 85 & 14 \\
\hline \multicolumn{3}{|l|}{ Insurance Status } \\
\hline Medicaid & 145 & 24 \\
\hline Private/HMO & 420 & 69 \\
\hline Self-pay/no charge & \# & 1 \\
\hline Other & 43 & 7 \\
\hline \multicolumn{3}{|l|}{ Household income by quartile* } \\
\hline First quartile $(\$ 1-24,999)$ & 100 & 16 \\
\hline Second quartile $(\$ 25,000-34,999)$ & 138 & 23 \\
\hline Third quartile $(\$ 35,000-44,999)$ & 164 & 27 \\
\hline Fourth quartile $(\$ 45,000+)$ & 201 & 33 \\
\hline \multicolumn{3}{|l|}{ Bed size of hospital ${ }^{\star \star}$} \\
\hline Small & 30 & 5 \\
\hline Medium & 146 & 24 \\
\hline Large & 407 & 67 \\
\hline \multicolumn{3}{|l|}{ Hospital location/teaching status } \\
\hline Rural & 12 & 2 \\
\hline Urban non-teaching & 43 & 7 \\
\hline Urban teaching & 527 & 87 \\
\hline
\end{tabular}

Table 1 (continued)
Table 1 (continued)

\begin{tabular}{|c|c|c|}
\hline \multirow{2}{*}{ Characteristic } & \multicolumn{2}{|c|}{ Total } \\
\hline & $\mathrm{N}$ & $\%$ \\
\hline \multicolumn{3}{|l|}{ Surgical procedure } \\
\hline Total abdominal colectomy & 413 & 68 \\
\hline Total colectomy with proctectomy & 196 & 32 \\
\hline \multicolumn{3}{|l|}{ Surgical technique } \\
\hline Laparoscopic & 377 & 62 \\
\hline Open & 232 & 38 \\
\hline
\end{tabular}

\section{Results}

A nationwide total of 609 pediatric patients were identified who underwent surgical treatment for UC during the study period. The two surgical procedures performed included total abdominal colectomy $(\mathrm{n}=413 ; 68 \%)$ and total colectomy with proctectomy ( $\mathrm{n}=196 ; 32 \%)$. The cohort was evenly distributed with respect to gender and elective $v s$. non-elective admissions. The majority of patients were Caucasian $(\mathrm{n}=399 ; 66 \%)$, had private/HMO insurance $(n=420,69 \%)$, and were treated in urban teaching hospitals $(\mathrm{n}=527 ; 87 \%)$. There was also a predilection to laparoscopic technique $(\mathrm{n}=377 ; 62 \%)$ in the cohort compared to open procedures. Additional demographic data is illustrated in Table 1.

Several variables in the cohort were found to be significantly associated with surgical approach and are demonstrated in Table 2. Patients were more likely to have laparoscopic operations at urban teaching hospitals than at rural or urban non-teaching hospitals $(65 \% v s$. $38 \%, \mathrm{P}<0.001)$. Although rates of laparoscopic surgery were higher in patients with private/HMO and Medicaid, patients with private insurance had a significantly lower rate of open procedures versus patients with Medicaid (35\% vs. $46 \% ; \mathrm{P}=0.02)$. Finally, no significant difference in the rates of open or laparoscopic procedures was detected by race. However, rates of laparoscopic procedures were still slightly higher in Caucasians (64\% vs. 55\% in African Americans) and it may be that this difference did not approach statistical 
Table 2 Predictors of laparoscopic vs. open

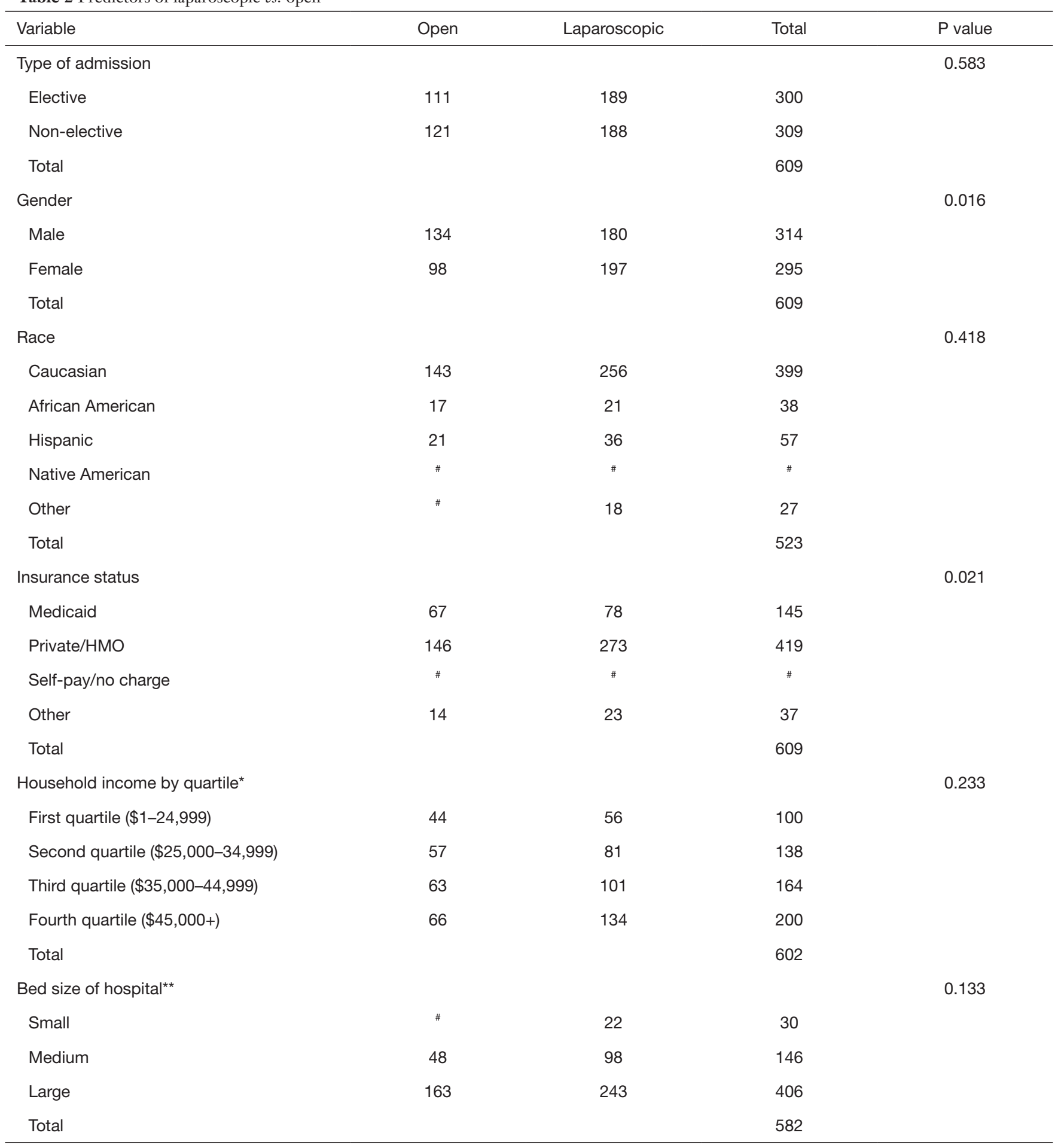

Table 2 (continued) 
Table 2 (continued)

\begin{tabular}{|c|c|c|c|c|}
\hline Variable & Open & Laparoscopic & Total & $P$ value \\
\hline Rural & $\#$ & $\#$ & 13 & \\
\hline Urban non-teaching & 28 & 15 & 43 & \\
\hline Urban teaching & 185 & 342 & 527 & \\
\hline Region of hospital & & & & 0.179 \\
\hline Northeast & 36 & 58 & 94 & \\
\hline Midwest & 77 & 128 & 205 & \\
\hline South & 67 & 83 & 150 & \\
\hline
\end{tabular}

*, actual dollar amounts are approximations across all year ranges and are adjusted yearly for inflation; **, definition varies slightly by region; \#, results censored due to KID reporting requirement for low number of cases.

significance due to the small sample size of non-white races. The remainder of the associated variables are shown in Table 2 .

In patients who underwent total abdominal colectomy without proctectomy, an open procedure was associated with a significantly longer median length of stay (14 vs. 11 days; $\mathrm{P}<0.01)$. The open approach was also significantly associated with increased risk for fluid and electrolyte disorders ( $40 \%$ vs. $28 \%$ ), GI disorders (16\% vs. $7 \%$ ), coagulopathy ( $9 \%$ vs. $3 \%$ ), septicemia (18\% vs. $2 \%)$, wound dehiscence $(6 \%$ vs. $2 \%)$, and pneumonia $(5 \%$ vs. $1 \%)$ as demonstrated in Table 3 (all $\mathrm{P}<0.05$ ).

Multivariate analysis was then performed to compare all statistically significant complications against relevant predictive variables (Table 4). Patients who underwent total colectomy without proctectomy, had significantly increased risk for any complication with an open approach (OR 2.75; $\mathrm{P}<0.001)$. Similarly, non-elective admission also significantly increased the odds of experiencing a complication following any approach $(\mathrm{OR} 2.47 ; \mathrm{P}<0.001)$.

A similar analysis of patients who underwent total colectomy with proctectomy is displayed in Table 5 . Univariate analysis revealed that open procedures were significantly associated with an increased risk for GI disorders $(11 \%$ vs. $1 \%)$ as well as increased transfusion requirements $(25 \%$ vs. $7 \%)($ all $\mathrm{P}<0.005)$. There was a statistically significant association between septicemia and the laparoscopic approach but overall numbers were low. Multivariate analysis of this cohort also revealed that an open approach $(\mathrm{OR} 3.83 ; \mathrm{P}<0.001)$ and non-elective admissions (OR 3.91; $\mathrm{P}<0.001)$ were significantly associated with increased odds of complications following surgery as shown in Table 6.

\section{Discussion}

$\mathrm{UC}$ is a relatively common, chronic disease that is a cause of serious morbidity in the pediatric population. This retrospective analysis represents the largest multiinstitutional study to date which compares the outcomes of pediatric patients who underwent surgical treatment for UC. Additionally, while prior studies have demonstrated equivalency of the laparoscopic and open approaches, we have demonstrated for the first time that a laparoscopic approach to the treatment of this disease is associated with an improvement in post-surgical outcomes as well as shorter overall length of hospital stay in patients who undergo total colectomy without proctectomy.

It is estimated that over 150,000 children suffer from IBD in the United States alone (15). Although already a devastating diagnosis, the subpopulation diagnosed with UC often have significantly worse outcomes than their adult counterparts (16). Over $80 \%$ of all UC patients under 20 will be diagnosed with pancolitis and these patients often 
Table 3 Univariate analysis-total colectomy without proctectomy*

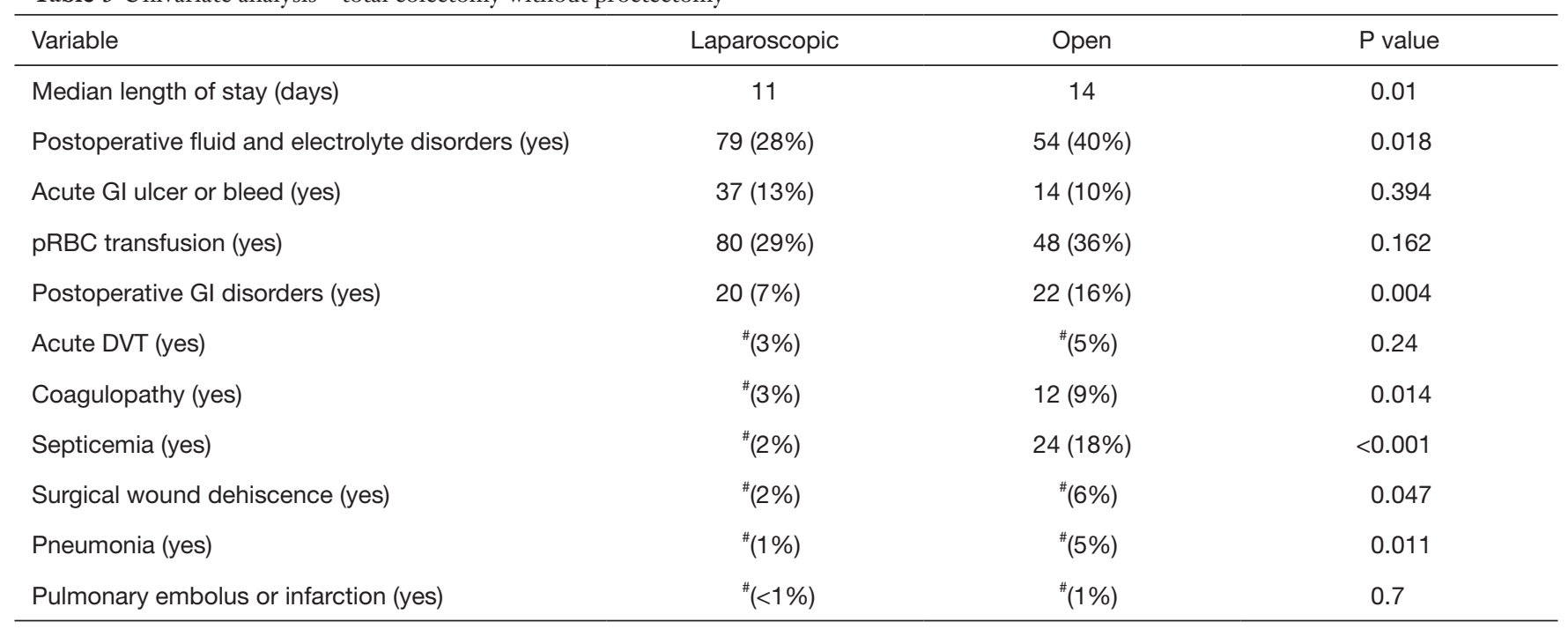

*, all percentages rounded to the nearest whole percent; ${ }^{*}$, results censored due to KID reporting requirement for low number of cases.

Table 4 Multivariate analysis-total colectomy without proctectomy

\begin{tabular}{lcc}
\hline Variable & Total colectomy & Odds ratio \\
\cline { 2 - 3 } & & \\
Surgical procedure & Total complications & 2.75 \\
Open & $46 \%(n=61)$ & \\
Laparoscopic & $23 \%(n=65)$ & \\
Admission status & & 2.47 \\
Non-elective & $37 \%(n=96)$ & \\
Elective & $19 \%(n=30)$ & $<0.001$ \\
\hline
\end{tabular}

require faster and further escalation of medical and surgical care than their adult counterparts (17). Due to the potential for severe complications from surgery the first line therapies for UC are primarily medical and include salicylates, antibiotics, steroids, and biologic agents (18).

However, surgical treatment of UC remains the cornerstone of therapy and nearly every patient diagnosed with UC will eventually require colectomy with ileal pouchanal anastomosis (19). Surgery for UC falls into two broad categories, elective procedures and non-elective procedures. Elective procedures (one, two, or three stage operations) are generally for curative intent and are indicated when the patient has been diagnosed with early malignant disease or when the patient has become steroid dependent for adequate symptom control. Emergent (two or three stage operations) procedures are performed for acute complications of the disease including toxic megacolon, perforation, and uncontrolled bleeding $(5,8)$. This dichotomy between indications for surgery was evident in our cohort as the majority of proctocolectomies in our patient population (74\%) were performed electively. Similarly, we observed that most colectomies $(63 \%)$ were performed in patients who were admitted non-electively indicating that a subtotal colectomy in our cohort was primarily used to manage the acute complications of UC.

It is interesting to note that the difference in outcomes between open and laparoscopic surgery was minimal in the patients who underwent total colectomy with proctectomy. In fact, complications in this group overall were much lower than what was seen in those patients who underwent 
Table 5 Univariate analysis - total proctocolectomy*

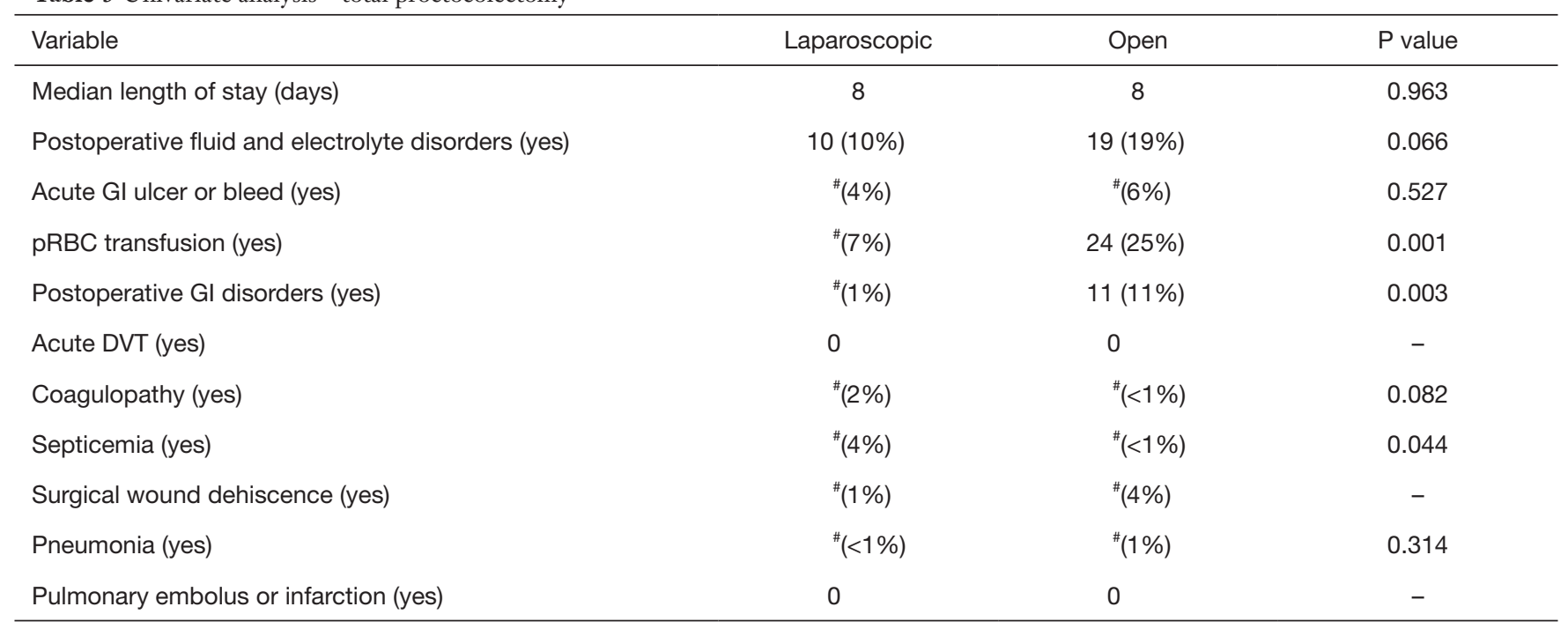

*, all percentages rounded to the nearest whole percent; ", results censored due to KID reporting requirement for low number of cases.

Table 6 Multivariate analysis-total proctocolectomy

\begin{tabular}{lccc}
\hline \multirow{2}{*}{ Variable } & \multicolumn{2}{c}{ Total proctocolectomy } \\
\cline { 2 - 4 } & $\begin{array}{c}\text { Total } \\
\text { complications }\end{array}$ & Odds ratio & P value \\
\hline Surgical procedure & $33 \%(n=32)$ & 3.83 & $<0.001$ \\
Open & $11 \%(n=11)$ & & \\
Laparoscopic & & & \\
Admission status & & 3.91 & $<0.001$ \\
Non-elective & $41 \%(n=21)$ & & \\
Elective & $15 \%(n=22)$ & & \\
\hline
\end{tabular}

colectomy alone. The reason for this is likely due to the majority of colectomies being performed for patients who were admitted due to non-elective indications while colectomy with proctectomy was reserved for patients with elective admissions. We were somewhat surprised to note that most colectomies performed for patients who were admitted non-electively in our cohort were performed laparoscopically $(63 \%)$. This may indicate that surgeon proficiency and comfort with laparoscopic techniques has increased dramatically over the preceding decade and these techniques are now being used even in the treatment of complex cases. In fact, the majority of improvements in patient outcomes seen with laparoscopic technique occurred in the cohort of non-elective admissions. Therefore, our analysis appears to agree with recent literature which indicates that the laparoscopic approach is appropriate as first line surgical therapy in even the sickest patients and should not be exclusively reserved for elective procedures (20).

In addition to the observed reduction in complications, laparoscopic total colectomies in our cohort were also significantly associated with a shorter median length of stay than open total colectomies. Length of stay is commonly used as a surrogate for cost and we did indeed observe a trend towards lower total cost of care in the laparoscopic group although it did not attain statistical significance due to high variability (results not shown). Historically, studies have cited the longer operative times of laparoscopic surgery as a potential drawback of this approach (21). As KID does not record procedure times, we were unable to comment on this in our analysis. However, more recent studies have been published which have found that, as surgeon experience increases, operative times for laparoscopic procedures have improved and become comparable to times seen in open procedures (22).

Our findings are not without limitations. KID is a strictly regulated source of pediatric inpatient information sampled across participating US hospitals. Still, there are possibilities that heterogeneity may exist in the data entry process due to institutional variability. Additionally, the analysis is retrospective, and as such we are limited to information coded in ICD-9-CM classifications. Furthermore, KID is a database of admissions and as such does not have information on prior treatments or surgeries that the 
patients in our cohort may have received. This is significant as many UC patients have received steroids or biologics during the course of their treatment and prior studies have identified frequency and volume of steroid usage as well as recent use of anti-TNF $\alpha$ agents as being associated with worse outcomes following surgery $(23,24)$. Lastly, while admissions are categorized as elective or non-elective, KID does not have the granularity to determine how soon after admission a patient undergoes an operation. Therefore, it is impossible to determine the precise medical acuity of patients at the time of operation and admission status is instead used as a surrogate.

\section{Conclusions}

$\mathrm{UC}$ in the pediatric population is the cause of significant morbidity and mandates eventual surgical intervention in all patients. We observed significantly lower length of stay in patients treated with laparoscopy compared to those who had an open procedure. The laparoscopic approach and elective surgery were both associated with a significantly lower rate of surgical complications in patients undergoing total colectomy with or without proctocolectomy. These findings demonstrate that laparoscopic technique is associated with more favorable outcomes compared to open total colectomy with or without proctectomy in pediatric UC patients.

\section{Acknowledgments}

Funding: None.

\section{Footnote}

Provenance and Peer Review: This article was commissioned by the editorial office, Translational Gastroenterology and Hepatology for the series "Current Topics in Pediatric General Surgery". The article has undergone external peer review.

Reporting Checklist: The authors have completed the STROBE reporting checklist. Available at http://dx. doi. org/10. 21037/tgh-20-189

Conflicts of Interest: All authors have completed the ICMJE uniform disclosure form (available at http://dx. doi. org/10. 21037/tgh-20-189). The series "Current Topics in Pediatric General Surgery" was commissioned by the editorial office without any funding or sponsorship. Dr. EAP served as the unpaid Guest Editor of the series and serves as an unpaid editorial board member of Translational Gastroenterology and Hepatology from Jan 2019 to Dec 2020. Dr. SP served as the unpaid Guest Editor of the series. The authors have no other conflicts of interest to declare.

Ethics Statement: The authors are accountable for all aspects of the work in ensuring that questions related to the accuracy or integrity of any part of the work are appropriately investigated and resolved. The study was conducted in accordance with the Declaration of Helsinki (as revised in 2013). The Institutional Review Board of the University of Miami waived the requirement for approval of this study since the KID contains de-identified, publicly available data.

Open Access Statement: This is an Open Access article distributed in accordance with the Creative Commons Attribution-NonCommercial-NoDerivs 4.0 International License (CC BY-NC-ND 4.0), which permits the noncommercial replication and distribution of the article with the strict proviso that no changes or edits are made and the original work is properly cited (including links to both the formal publication through the relevant DOI and the license). See: https://creativecommons.org/licenses/by-nc-nd/4.0/.

\section{References}

1. Cabrera JM, Sato TT. Medical and Surgical Management of Pediatric Ulcerative Colitis. Clin Colon Rectal Surg 2018;31:71-9.

2. Drews JD, Onwuka EA, Fisher JG, et al. Complications after proctocolectomy and ileal pouch-anal anastomosis in pediatric patients: A systematic review. J Pediatr Surg 2019;54:1331-9.

3. Cosnes J, Gower-Rousseau C, Seksik P, et al. Epidemiology and natural history of inflammatory bowel diseases. Gastroenterology 2011;140:1785-94.

4. Cuffari C. Inflammatory bowel disease in children: a pediatrician's perspective. Minerva Pediatr 2006;58:139-57.

5. Feuerstein JD, Cheifetz AS. Ulcerative colitis: epidemiology, diagnosis, and management. Mayo Clin Proc 2014;89:1553-63.

6. Mahida JB, Asti L, Deans KJ, et al. Laparoscopic bowel resection for pediatric inflammatory bowel disease. J Surg Res 2015;199:130-6. 
7. Yu YR, Rodriguez JR. Clinical presentation of Crohn's, ulcerative colitis, and indeterminate colitis: Symptoms, extraintestinal manifestations, and disease phenotypes. Semin Pediatr Surg 2017;26:349-55.

8. Siow VS, Bhatt R, Mollen KP. Management of acute severe ulcerative colitis in children. Semin Pediatr Surg 2017;26:367-72.

9. Tan Tanny SP, Yoo M, Hutson JM, et al. Current surgical practice in pediatric ulcerative colitis: A systematic review. J Pediatr Surg 2019;54:1324-30.

10. Page AE, Sashittal SG, Chatzizacharias NA, et al. The role of laparoscopic surgery in the management of children and adolescents with inflammatory bowel disease. Medicine (Baltimore) 2015;94:e874.

11. Mattioli G, Pini-Prato A, Barabino A, et al. Laparoscopic approach for children with inflammatory bowel diseases. Pediatr Surg Int 2011;27:839-46.

12. Linden BC, Bairdain S, Zurakowski D, et al. Comparison of laparoscopic-assisted and open total proctocolectomy and ileal pouch anal anastomosis in children and adolescents. J Pediatr Surg 2013;48:1546-50.

13. (HCUP) HCaUP. KID Database Documentation. Volume 2019. Rockville, MD: Agency for Healthcare Research and Quality, 2018.

14. U.S Census Bureau 2019. National Intercensal Datasets. Available online: https://www.census.gov/programssurveys/popest/data/data-sets.html

15. Rosen MJ, Dhawan A, Saeed SA. Inflammatory Bowel Disease in Children and Adolescents. JAMA Pediatr 2015;169:1053-60.

16. Bradley GM, Oliva-Hemker M. Pediatric ulcerative colitis:

doi: $10.21037 / \operatorname{tgh}-20-189$

Cite this article as: Willobee BA, Nguyen JA, Ferrantella A, Quiroz HJ, Hogan AR, Brady AC, Pandya S, Langshaw AH, Sola JE, Thorson CM, Perez EA. A retrospective comparison of outcomes for open $v s$. laparoscopic surgical techniques in pediatric ulcerative colitis. Transl Gastroenterol Hepatol 2021;6:41. current treatment approaches including role of infliximab. Biologics 2012;6:125-34.

17. Uchida K, Araki T, Toiyama Y, et al. Preoperative steroidrelated complications in Japanese pediatric patients with ulcerative colitis. Dis Colon Rectum 2006;49:74-9.

18. Lee WJ, Briars L, Lee TA, et al. Top-down Versus Stepup Prescribing Strategies for Tumor Necrosis Factor Alpha Inhibitors in Children and Young Adults with Inflammatory Bowel Disease. Inflamm Bowel Dis 2016;22:2410-7.

19. Dayan B, Turner D. Role of surgery in severe ulcerative colitis in the era of medical rescue therapy. World journal of gastroenterology 2012;18:3833-8.

20. Sauerland S, Agresta F, Bergamaschi R, et al. Laparoscopy for abdominal emergencies: evidence-based guidelines of the European Association for Endoscopic Surgery. Surg Endosc 2006;20:14-29.

21. Fleming FJ, Francone TD, Kim MJ, et al. A laparoscopic approach does reduce short-term complications in patients undergoing ileal pouch-anal anastomosis. Dis Colon Rectum 2011;54:176-82.

22. McKenna NP, Potter DD, Bews KA, et al. Ileal-pouch anal anastomosis in pediatric NSQIP: Does a laparoscopic approach reduce complications and length of stay? J Pediatr Surg 2019;54:112-7.

23. Koike Y, Uchida K, Inoue M, et al. Predictors for Pouchitis After Ileal Pouch-Anal Anastomosis for Pediatric-Onset Ulcerative Colitis. J Surg Res 2019;238:72-8.

24. Billioud V, Ford AC, Tedesco ED, et al. Preoperative use of anti-TNF therapy and postoperative complications in inflammatory bowel diseases: a meta-analysis. J Crohns Colitis 2013;7:853-67. 